Nature 589: 567-571. DOI: https://doi.org/10.1038/s41586-020-03173-9

\title{
Half a century of global decline in oceanic sharks and rays
}

Authors: Nathan Pacoureau*,1, Cassandra L. Rigby ${ }^{2}$, Peter M. Kyne ${ }^{3}$, Richard B. Sherley*,4, Henning Winker ${ }^{5,6}$, John K. Carlson ${ }^{7}$, Sonja V. Fordham ${ }^{8}$, Rodrigo Barreto ${ }^{9}$, Daniel Fernando $^{10}$, Malcolm P. Francis ${ }^{11}$, Rima W. Jabado ${ }^{12}$, Katelyn B. Herman ${ }^{13}$, Kwang-Ming Liu $^{14}$, Andrea D. Marshall ${ }^{15}$, Riley A. Pollom ${ }^{1}$, Evgeny V. Romanov ${ }^{16}$, Colin A. Simpfendorfer ${ }^{2}$, Jamie S. Yin ${ }^{1,17}$, Holly K. Kindsvater ${ }^{18}$ and Nicholas K. Dulvy ${ }^{1}$

\section{Affiliations:}

${ }^{1}$ Department of Biological Sciences, Earth to Ocean Research Group, Simon Fraser University, Burnaby, British Columbia, Canada.

${ }^{2}$ Centre for Sustainable Tropical Fisheries and Aquaculture \& College of Science and Engineering, James Cook University, Queensland, Australia.

${ }^{3}$ Research Institute for the Environment and Livelihoods, Charles Darwin University, Darwin, Northern Territory, Australia.

${ }^{4}$ Centre for Ecology and Conservation, College of Life and Environmental Sciences, University of Exeter, Penryn Campus, Cornwall, United-Kingdom.

${ }^{5}$ Joint Research Centre (JRC), European Commission, Ispra, Italy.

${ }^{6}$ Department of Environment, Forestry and Fisheries, Cape Town, South Africa.

${ }^{7}$ NOAA National Marine Fisheries Service, Southeast Fisheries Science Center, Panama City Laboratory, Panama City, FL, United States of America.

${ }^{8}$ Shark Advocates International, The Ocean Foundation, Washington, DC, United States of America.

${ }^{9}$ Centro Nacional de Pesquisa e Conservação da Biodiversidade Marinha do Sudeste e Sul do Brasil (CEPSUL), Instituto Chico Mendes de Conservação da Biodiversidade (ICMBio), Itajaí, SC, Brazil.

${ }^{10}$ Blue Resources Trust, Colombo, Sri Lanka.

${ }^{11}$ National Institute of Water and Atmospheric Research, Wellington, New Zealand.

${ }^{12}$ Elasmo Project, Dubai, United Arab Emirates.

${ }^{13}$ Georgia Aquarium, Atlanta, GA, United States of America.

${ }^{14}$ Institute of Marine Affairs and Resource Management, George Chen Shark Research

Center, National Taiwan Ocean University, Center of Excellence for the Oceans, National

Taiwan Ocean University, Taiwan.

${ }^{15}$ Marine Megafauna Foundation, Truckee, CA, United States of America.

${ }^{16}$ CAP RUN — CITEB, Le Port, Île de la Réunion, France.

${ }^{17}$ Department of Ecology, Evolution, and Natural Resources, Rutgers University, New Brunswick, NJ, United States of America.

${ }^{18}$ Department of Fish and Wildlife Conservation, Virginia Polytechnic Institute and State University, Blacksburg, VA, United States of America. 
40

Summary: Overfishing is the primary cause of marine defaunation, yet individual species' declines and rising extinction risk are difficult to measure, particularly for the largest predators found in the high seas ${ }^{1-3}$. We calculate two well-established indicators to track progress towards Aichi Biodiversity Targets and Sustainable Development Goals $^{4,5}$ : the Living Planet Index (a measure of changes in abundance aggregating 57 abundance time-series for 18 oceanic shark and ray species), and the Red List Index (a measure of change in extinction risk calculated for all 31 oceanic species). We find that, since 1970, the global abundance of oceanic sharks and rays has declined by $71 \%$ due to an 18-fold increase in Relative Fishing Pressure. This depletion elevated global extinction risk to the point where three-quarters of this functionally important assemblage are threatened with extinction. Strict prohibitions and precautionary science-based catch limits are urgently needed to avert population collapse ${ }^{6,7}$, avoid disruption of ecological function, and promote species recovery ${ }^{8,9}$.

Over the United Nations 'Decade of Biodiversity' from 2011-2020, governments committed to improve human well-being and food security by safeguarding ecosystem services and halting biodiversity $\operatorname{loss}^{10}$. The Sustainable Development Goals, adopted by all United Nations Member States, and the 20 Aichi Biodiversity Targets of the Convention on Biological Diversity, provide a framework to track progress towards the 2020 deadline $e^{4,5,10}$. Seafood sustainability is an integral part of these commitments, and wild capture fisheries are essential nutritional and economic resources for millions of people globally ${ }^{11,12}$. Yet beneath the ocean surface, it is difficult to assess changes in the state of biodiversity and ecosystem structure, function, and services ${ }^{13}$. 
65 Elasmobranchs (sharks and rays, hereafter 'sharks') offer a unique window into the state of

66 the oceans. Sharks are one of the most evolutionarily distinct and functionally diverse

67 vertebrate radiations ${ }^{14,15}$. The first International Union for Conservation of Nature (IUCN)

68 global assessment estimated that one-quarter of sharks were Threatened with extinction

69 (classified as Critically Endangered, Endangered, or Vulnerable according to IUCN Red List

70 criteria $)^{16}$, making sharks the most threatened vertebrate lineage after amphibians ${ }^{16-18}$. Long

71 generation times and low intrinsic population growth rates of many sharks make them

72 inherently susceptible to overexploitation ${ }^{1,7,19}$. Globally, sharks are landed for their meat,

73 fins, gill plates, and liver oil ${ }^{20,21}$ and catches rose to an estimated peak of 63-273 million

74 individuals in the early 2000 s before declining due to overfishing ${ }^{6}$. The first warnings of the

75 dire status of sharks were based on boom and bust catch patterns and rising international

76 trade in shark fins ${ }^{22,23}$. Subsequently, serious declines in many oceanic and coastal shark

77 populations were documented, both in the Gulf of Mexico and Northwest Atlantic ${ }^{24,25}$, and

78 also in South Africa ${ }^{26}$ and Australia ${ }^{27}$. Shark population assessments for many other regions

79 have since become increasingly robust ${ }^{8,28,29}$. Until now, however, these have not yet been

80 synthesised to provide a global perspective on shark population trends.

81 Here, we calculate for oceanic sharks two Biodiversity Indicators established by the

82 Convention on Biological Diversity: the Living Planet Index (LPI) ${ }^{5,30}$ on global population

83 changes since 1970 and the Red List Index (RLI) ${ }^{5,31}$, which tracks changes in the relative

84 extinction risk of taxa. These indicators quantify progress toward Aichi Targets 6 (manage

85 marine resources for sustainability) and 12 (prevent extinction), and UN Sustainable

86 Development Goal 14 (conserve and sustainably use the oceans). First, we used a Bayesian

87 state-space framework ${ }^{32,33}$ to estimate trends in relative abundance of 18 species from 57

88 time-series compiled and reviewed at an expert workshop convened by the IUCN Species

89 Survival Commission's Shark Specialist Group (IUCN SSC SSG). Using these trends, we 
90 calculated the global LPI for oceanic sharks from the reference year 1970 (which was set at

91 1) to 2018 - and then extrapolated each time-series to 2020 to encompass the Aichi Target

92 assessment year - by hierarchically aggregating the annual rates of change from each time-

93 series for a species by region, then globally (see Extended Data Figure [EDF] 1 and 2a).

94 Second, we combined a retrospective Red List assessment (1980) with two recent

95 assessments ( 2005 and 2018) from the IUCN Red List of Threatened Species for all 31

96 species of oceanic sharks to build the RLI (see EDF 1). The RLI provides standardized

97 assessments of species' extinction risk, comparable across taxa, that is particularly useful

98 when robust trend data are missing. Comparing the RLI over time, among different taxa,

99 reveals the common trends in extinction risk among groups, despite differences in habitat, life

100 history, and threats. Such cross-taxon comparisons are useful to ensure appropriate allocation

101 of global conservation resources across terrestrial, freshwater, and marine biomes.

102 Finally, we develop three lines of evidence to attribute decreasing abundance (shown by the

103 LPI) and rising extinction risk (shown by the RLI) of oceanic sharks to overfishing: (i)

104 increasing Relative Fishing Pressure over time (measured as changes in catch relative to the

105 changes in the LPI), (ii) increasing proportion, over time, of oceanic sharks that are

106 overfished below biomass or abundance levels that could produce Maximum Sustainable

107 Yield (MSY, the equilibrium state of the exploited population sustaining the greatest yield

108 [catch] over long time periods ${ }^{34}$ ), and (iii) the near-absence of significant threats other than

109 fishing reported in each species' IUCN Red List assessment.

\section{Declining abundance index}

112 We find that, globally, abundance of oceanic sharks declined by $71.1 \%(95 \%$ credible

113 interval [CI]: 63.2-78.4\%; Fig. 1) from 1970 to 2018, at a steady rate averaging $18.2 \%$ per

114 decade (see EDF 2c). Over the half-century from 1970-2020, the projected LPI estimates that 
115 abundance declined by $70.1 \%$ (CI: $62.8-77.2 \%$, see EDF $2 \mathrm{~b}$ ). The declining trend of the LPI

116 trajectory is robust to the exclusion of any individual species (EDF 3). There are three

117 reasons why the true abundance trend index values are likely to be lower (and calculated

118 percent declines worse) than estimated here (see Supplementary Discussion 1): (i) fishing

119 levels were already unsustainable half a century ago, (ii) unreported catches (including

120 discards) are not included in our time-series, and (iii) traditional stock assessments could

121 underestimate fishing mortality.

122 The global trend index can be disaggregated into trajectories for each ocean and species, as

123 well as for functional groups with similar ecological or life-history traits. In the Atlantic

124 Ocean, following a long period of decline since 1970, abundance began to stabilize at low

125 levels after 2000 (overall decline of 46.1\%; CI: 30.7-61.1\%; Fig. 2a). In the Pacific Ocean,

126 abundance decreased steeply prior to 1990 , and then declined at a slower rate (overall decline

127 of $67.0 \%$; CI: 53.6-79.4\%; Fig. 2c). In the Indian Ocean, shark abundances have declined

128 steeply since 1970 (overall decline of 84.7\%; CI: 75.9-92.1\%; Fig. 2b). Despite more

129 resilient life histories, tropical sharks declined more steeply than temperate species (overall

130 declines of $87.8 \%$; CI: $79.8-94.3 \%$ versus $40.9 \%$; CI: $30.4-50.5 \%$, Fig. 2 d). Overfishing of

131 sharks followed a classic pattern of serial depletion, starting with the largest species, which

132 dropped steeply prior to the 1980 s, followed by declines of medium-sized species and

133 eventually relatively small species (including some devil rays, Mobula spp.; Fig. 2e). Long

134 lived, late-maturing species initially declined faster than those with shorter generation times,

135 but two of these species (White Shark Carcharodon carcharias and Porbeagle Lamna nasus)

136 have shown signs of population rebuilding since the early 2000s (Fig. 2f; EDF 7). All species,

137 apart from the Smooth Hammerhead (Sphyrna zygaena), decreased in abundance over the last

138 half-century (Fig. 2g). Devil ray abundance has declined by at least $85 \%$ in the past 15 years

139 in the Southwest Indian Ocean (Fig. 2g). Although sparse, the available data for devil rays are 
140 representative of the repeated, rapid depletions and local extinctions suspected due to

141 overfishing driven by target fisheries in many parts of their historical range (see

142 Supplementary Discussion 2).

\section{$143 \quad$ Rising extinction risk}

144 For all 31 oceanic shark species, the risk of extinction, indicated by IUCN Red List category,

145 has substantially worsened since 1980 . The RLI declined from a retrospective estimate of

1460.86 (range: $0.74-0.90$ ) in 1980 to 0.56 in 2018, comparable to cycads (palm-like plants), the

147 most threatened group of completely assessed species on Earth ${ }^{35}$ (Fig. 3a). We estimate that

148 in 1980, two-thirds $(n=20)$ of oceanic shark species fell into the IUCN Red List category of

149 Least Concern, and only nine were Threatened. The Basking Shark (Cetorhinus maximus)

150 was the only species retrospectively classified as Endangered. More than three-quarters

$151(n=24)$ of these species are Threatened now based on steep population reductions (IUCN Red

152 List Criterion A). Some formerly abundant, wide-ranging sharks have declined so steeply that

153 they are now classified in the two highest IUCN Red List categories: three are Critically

154 Endangered (Oceanic Whitetip Shark Carcharhinus longimanus, Scalloped and Great

155 Hammerhead Sphyrna lewini and S. mokarran), and four are Endangered (Pelagic Thresher

156 Alopias pelagicus, Dusky Shark Carcharhinus obscurus, Shortfin and Longfin Mako Isurus

157 oxyrinchus and I. paucus; Fig. 3b). In total, half (15 of 31) of oceanic shark species are now

158 Critically Endangered ( $n=3 ; \geq 80 \%$ population reduction over three generations) or

159 Endangered $(n=12 ; 50-79 \%$ population reduction $)$.

160 Attributing declines and rising extinction risk to overfishing

161 We attribute oceanic shark population declines and elevated extinction risk to overfishing

162 based on three lines of evidence. First, the last half-century has seen more than a two-fold

163 increase in fishing with longlines and seine nets, the gears that catch the most oceanic

$164 \operatorname{sharks}^{36}$ (Fig. 4a; black lines; data corrected for technological creep, see Supplementary 
165 Methods 1). Concomitantly, oceanic shark catch has risen three-fold since 1970 (Fig. 4a; grey

166 line and polygons), resulting in an 18-fold increase in Relative Fishing Pressure (Fig 4b).

167 This correlation suggests fishing drove declines in abundance with a striking breakpoint in

1681990 that we hypothesize coincides with increasing retention of sharks to meet new market

169 demands (specifically for fins) ${ }^{37}$ (Fig. 4c). Second, the role of fisheries in driving declines is

170 thoroughly addressed in the growing number of robust fisheries stock assessments (EDF 9b).

171 The declining LPI is consistent with a rising proportion of populations and species assessed

172 as overfished over time (21\%; Fig. 4d); 6 of the 8 assessed species and over half of the

173 populations (9 of 15) are below MSY (EDF 9c). Third, we compiled the causes of declines

174 reported in Red List assessments, which are classified into 11 categories ranging from

175 'Human Intrusions and Disturbance', to 'Climate and Severe Weather' ${ }^{38}$. While there are

176 numerous pressures acting on sharks, every Red List assessment for the 31 oceanic sharks

177 concluded that the major threat was 'Biological Resource Use' and, more specifically,

178 'Fishing and Harvesting Aquatic Resources'. Other threats are reported for only two species

179 (EDF 10).

180

\section{Discussion}

182 We document an alarming, ongoing, worldwide decline of oceanic shark populations across

183 the world's largest ecosystem over the past half-century, resulting in an unprecedented

184 increase in the risk of extinction of these species. The tremendous increase in Relative

185 Fishing Pressure is mirrored by the general consistency in the rate and extent of declines

186 across species of differing body sizes and generation times. The low reproductive output of

187 these slow-growing species is clearly no match for the intense fishing pressure they currently

188 encounter. 
189 Overfishing of oceanic shark populations has far outpaced the implementation of fisheries

190 management and trade regulations ${ }^{39}$. Despite great strides in conservation commitments in

191 recent decades, relatively few countries impose catch limits specific to oceanic sharks, and

192 fewer still can demonstrate population rebuilding or sustainable fisheries for these species.

193 Obligations under international wildlife treaties $\left(\mathrm{see}^{7}\right)$ to prohibit retention or restrict

194 international trade of select species have not yet been effectively implemented ${ }^{40}$. The world's

195 four major Regional Fishery Management Organizations focused on tunas (tRFMOs) have, to

196 varying degrees, prohibited retention of inherently sensitive oceanic shark species that are

197 also of relatively low value to the associated pelagic fisheries. However, tRFMOs' efforts to

198 manage sharks using Ecosystem-Based Fisheries Management have been inadequate with

199 respect to scientific advice and implementation ${ }^{41,42}$ (see Supplementary Discussion 3).

200 There are some encouraging findings. We note that the White Shark historically declined by

201 an estimated $70 \%$ worldwide over the last half-century, but is now recovering in several

202 regions, aided by retention bans ${ }^{43}$. Hammerhead shark populations are rebuilding in the

203 Northwest Atlantic, owing to strictly enforced quotas throughout their U.S. range. The Blue

204 Shark has declined less than other species, despite being reported to be at significantly greater

205 risk due to its high distributional overlap with heavily fished areas ${ }^{44}$. This is likely due to its

206 relatively high reproductive rate (compared to other pelagic sharks), but nevertheless its

207 management is warranted on a global scale as market interest and targeted fishing increase. It

208 is possible to reverse shark population declines, even for slow-growing species, if

209 precautionary, science-based management is implemented throughout a species' range ${ }^{8,45}$

210 before depletion reaches a point of no return.

211 We can use IUCN Red List status and trends as a heuristic to guide the conservation priorities

212 of countries with limited capacity to assess, manage, and conserve oceanic species. This

213 guidance will be less relevant to nations with the capacity to undertake stock assessments and 
214 ensure compliance with management ${ }^{8}$, reflecting that a species' global Red List Status and

215 local status may differ. It has been previously recommended that sharks assessed globally as

216 Near Threatened or even some assessed as Vulnerable may still be able to sustain modest

217 levels of fishing, if managed immediately and carefully throughout their range ${ }^{7,16}$. Species

218 classified as Critically Endangered or Endangered cannot support fisheries. In these cases,

219 policy recommendations based on stock assessments or on global Red List Status will be

220 congruent $^{46}$; strict measures to prohibit landings and minimize bycatch mortality (by avoiding

221 hotspots, modifying gear, and improving release practices) are urgently needed to halt

222 declines and rebuild populations.

223 The ecosystem consequences of oceanic shark declines are uncertain because of the

224 complexity and scale of marine food webs ${ }^{47}$. Nevertheless, profound effects of depleting

225 predatory species are becoming apparent. For example, the decline of predatory sharks and

226 tunas is associated with increases in mesopredators, including teleosts and smaller-bodied

227 shark species ${ }^{48}$, indicating fundamental functional changes to marine food webs ${ }^{15}$. Of further

228 concern is the associated threat to food security and income in many poor and developing

229 nations $^{7}$, many of which have fished sharks for generations ${ }^{49}$. Alternative livelihood and

230 income options are needed to ease transitions to sustainability.

\section{Conclusion}

233 We demonstrate that — despite ranging farther from land than most species — oceanic

234 sharks are exceptionally threatened by overexploitation. It is clear that the Sustainable

235 Development Goals and specific Aichi targets (to reverse population declines and use marine

236 resources sustainably) have not been met by 2020 for these species. Action is needed

237 immediately to prevent shark population collapses and myriad negative consequences for

238 associated economic and ecological systems. Specifically, there is a clear and urgent need for 
239 governments to adopt, implement, and enforce - at domestic and regional levels - science-

240 based catch limits for oceanic sharks that are capable of supporting sustainable fisheries, and

241 retention prohibitions, along with bycatch mitigation, for the others ${ }^{7,8}$. These steps are

242 imperative for long-term sustainability, including potentially increased catch once

243 populations are rebuilt ${ }^{9,50}$, and a brighter future for some of the most iconic and functionally

244 important animals in our oceans.

\section{References:}

248 1. Dulvy, N. K. et al. You can swim but you can't hide: the global status and conservation of oceanic pelagic

249 sharks and rays. Aquat. Conserv. Mar. Freshw. Ecosyst. 18, 459-482 (2008).

250 2. Webb, T. J. \& Mindel, B. L. Global patterns of extinction risk in marine and non-marine systems. Curr. Biol.

$25125,506-511(2015)$.

252 3. McCauley, D. J. et al. Marine defaunation: Animal loss in the global ocean. Science 347, 1255641 (2015).

253 4. Tittensor, D. P. et al. A mid-term analysis of progress toward international biodiversity targets. Science 346, $254 \quad 241-244(2014)$.

255 5. Butchart, S. H. et al. Global biodiversity: indicators of recent declines. Science 328, 1164-1168 (2010).

256 6. Davidson, L. N., Krawchuk, M. A. \& Dulvy, N. K. Why have global shark and ray landings declined:

257 improved management or overfishing? Fish Fish. 17, 438-458 (2016).

258 7. Dulvy, N. K. et al. Challenges and priorities in shark and ray conservation. Curr. Biol. 27, R565-R572 (2017).

259 8. Simpfendorfer, C. A. \& Dulvy, N. K. Bright spots of sustainable shark fishing. Curr. Biol. 27, R97-R98 (2017).

260 9. Sumaila, U. R. et al. Benefits of rebuilding global marine fisheries outweigh costs. PloS One 7, e40542; 1-12 (2012).

261 10. Brooks, T. M. et al. Harnessing biodiversity and conservation knowledge products to track the Aichi Targets

262 and Sustainable Development Goals. Biodiversity 16, 157-174 (2015).

263 11. FAO. The State of World Fisheries and Aquaculture 2016. Contributing to food security and nutrition for

264 all. (Rome, 2016).

265 12. Hicks, C. C. et al. Harnessing global fisheries to tackle micronutrient deficiencies. Nature 574, 95-98 (2019). 

unknown. Annu. Rev. Environ. Resour. 37, 25-50 (2012).

14. Stein, R. W. et al. Global priorities for conserving the evolutionary history of sharks, rays and chimaeras. Nat. Ecol. Evol. 2, 288-298 (2018).

270 15. Pimiento, C. et al. Functional diversity of marine megafauna in the Anthropocene. Sci. Adv. 6, eaay7650 (2020).

16. Dulvy, N. K. et al. Extinction risk and conservation of the world's sharks and rays. elife 3, e00590 (2014).

17. Stuart, S. N. et al. Status and trends of amphibian declines and extinctions worldwide. Science 306, $1783-$ 1786 (2004).

18. Hoffmann, M. et al. The impact of conservation on the status of the world's vertebrates. Science 330, 15031509 (2010).

19. Pardo, S. A., Kindsvater, H. K., Reynolds, J. D. \& Dulvy, N. K. Maximum intrinsic rate of population increase in sharks, rays, and chimaeras: the importance of survival to maturity. Can. J. Fish. Aquat. Sci. 73, 1159-1163 (2016).

20. McClenachan, L., Cooper, A. B. \& Dulvy, N. K. Rethinking trade-driven extinction risk in marine and terrestrial megafauna. Curr. Biol. 26, 1640-1646 (2016).

21. Clarke, S. C. et al. Global estimates of shark catches using trade records from commercial markets. Ecol. Lett. 9, 1115-1126 (2006).

22. Brander, K. Disappearance of common skate Raia batis from Irish Sea. Nature 290, 48-49 (1981).

23. Manire, C. A. \& Gruber, S. H. Many sharks may be headed toward extinction. Conserv. Biol. 4, 10-11 (1990).

24. Southeast Data, Assessment, and Review (SEDAR). Update assessment to SEDAR 21. HMS Dusky Shark. (SEDAR, North Charleston, SC, USA, 2016).

25. International Commission for the Conservation of Atlantic Tunas (ICCAT). Report of the 2019 ICCAT Shortfin Mako Shark Stock Assessment Update Meeting. 41 (2019). off KwaZulu-Natal beaches, South Africa, 1978-2003. Mar. Freshw. Res. 57, 225-240 (2006). half century. Commun. Biol. 1, 1-11 (2018). 
29. Cortés, E. et al. Ecological risk assessment of pelagic sharks caught in Atlantic pelagic longline fisheries. Aquat. Living Resour. 23, 25-34 (2010).

30. Loh, J. et al. The Living Planet Index: using species population time series to track trends in biodiversity.

Philos. Trans. R. Soc. B Biol. Sci. 360, 289-295 (2005).

299 31. Butchart, S. H. et al. Improvements to the red list index. PloS One 2, e140 (2007).

300 32. Winker, H., Carvalho, F. \& Kapur, M. JABBA: Just Another Bayesian Biomass Assessment. Fish. Res. 204, $275-288$ (2018).

33. Sherley, R. B. et al. Estimating IUCN Red List population reduction: JARA-A decision support tool 303 applied to pelagic sharks. Conserv. Lett. e12688 (2020).

304 34. Punt, A. E. \& Smith, A. D. The gospel of maximum sustainable yield in fisheries management: birth, 305 crucifixion and reincarnation. Conserv. Exploit. Species 6, 41 (2001).

306 35. Marler, P. N. \& Marler, T. E. An assessment of Red List data for the Cycadales. Trop. Conserv. Sci. 8, $1114-1125$ (2015).

36. Anticamara, J. A., Watson, R., Gelchu, A. \& Pauly, D. Global fishing effort (1950-2010): trends, gaps, and implications. Fish. Res. 107, 131-136 (2011).

310 37. Vannuccini, S. Shark utilization, marketing, and trade. (Food \& Agriculture Org., 1999).

311 38. Salafsky, N. et al. A standard lexicon for biodiversity conservation: unified classifications of threats and 312 actions. Conserv. Biol. 22, 897-911 (2008).

313 39. Juan-Jordá, M. J., Mosqueira, I., Cooper, A. B., Freire, J. \& Dulvy, N. K. Global population trajectories of 314 tunas and their relatives. Proc. Natl. Acad. Sci. 108, 20650-20655 (2011).

315 40. Lawson, J. M. \& Fordham, F. Realizing the Potential of the Convention on Migratory Species to Conserve 316 Elasmobranchs. (2018).

317 41. Juan Jordá, M. J., Murua, H., Arrizabalaga, H., Dulvy, N. K. \& Restrepo, V. Report card on ecosystem based 318 fisheries management in tuna regional fisheries management organizations. Fish Fish. 19, 321-339 (2018).

319 42. Gilman, E., Passfield, K. \& Nakamura, K. Performance of regional fisheries management organizations:

320 ecosystem based governance of bycatch and discards. Fish Fish. 15, 327-351 (2014).

321 43. Curtis, T. H. et al. Seasonal distribution and historic trends in abundance of white sharks, Carcharodon

322 carcharias, in the western North Atlantic Ocean. PLoS One 9, e99240; 1-12 (2014).

323 44. Queiroz, N. et al. Global spatial risk assessment of sharks under the footprint of fisheries. Nature 572, 461$324466(2019)$ 
45. Peterson, C. D. et al. Preliminary recovery of coastal sharks in the south east United States. Fish Fish. 18, 845-859 (2017).

46. Jennings, S. Reporting and advising on the effects of fishing. Fish Fish. 8, 269-276 (2007).

47. Kitchell, J. F., Essington, T. E., Boggs, C. H., Schindler, D. E. \& Walters, C. J. The role of sharks and longline fisheries in a pelagic ecosystem of the central Pacific. Ecosystems 5, 202-216 (2002).

48. Polovina, J. J., Abecassis, M., Howell, E. A. \& Woodworth, P. Increases in the relative abundance of midtrophic level fishes concurrent with declines in apex predators in the subtropical North Pacific, 1996-2006. Fish. Bull. 107, 523-531 (2009).

49. Jabado, R. W. et al. Troubled waters: Threats and extinction risk of the sharks, rays and chimaeras of the Arabian Sea and adjacent waters. Fish Fish. 19, 1043-1062 (2018).

50. Costello, C. et al. Global fishery prospects under contrasting management regimes. Proc. Natl. Acad. Sci. 113, 5125-5129 (2016).

\section{Figure legend:}

339 Figure 1. Global Living Planet Index (LPI) for 18 oceanic sharks estimated from 1970 to

340 2018. The global percentage (\%) of decline was calculated from the posteriors of the LPI

341 around the final assessment year relative to the posteriors for 1970 . The black line denotes the

342 mean, the white lines the $95 \%$ credible intervals and the grey lines each iteration.

344 Figure 2. Living Planet Index for 18 oceanic sharks from 1970 to 2018 disaggregated by

345 Oceans (a, b, c), and the traits (d) geographical zone, (e) body size (maximum total length), (f)

346 generation time (GT), and (g) species (species' time-series are in Extended Data Figure 4 to

347 8). Lines denote the mean and shaded regions the $95 \%$ credible intervals.

349 Figure 3. (a) Global Red List Index (RLI) for the 31 oceanic shark species (black line)

350 estimated in 1980, 2005, and 2018, and for mammals, birds, amphibians, reef-forming corals, 351 and cycads (in grey), and global chondrichthyans (sharks, rays, and chimaeras; point labelled 
352 'Global sharks') ${ }^{16}$. The error bar denotes the uncertainty around the retrospective 1980 IUCN

353 status (see Methods). A RLI value of 1.0 equates to all species qualifying as Least Concern

354 (i.e., not expected to become Extinct in the near future), while a RLI value of 0 equates to all

355 species having gone Extinct. (b) Change in Red List status of oceanic sharks from 1980 to 3562018.

Figure 4. (a) Global catch data of 14 oceanic sharks and fishing effort of longline and seine gears. SAU: Sea Around Us project. FAO: Food and Agriculture Organization of the United and to their abundance from 1970 to 2014 . The black line denotes the mean, the white lines the $95 \%$ credible intervals and the grey lines each iteration. (c) Living Planet Index (LPI) as a

364 function of Relative Fishing Pressure (RFP, $n=14$ species) from 1970 (the initial state where

365 LPI and RFP $=1$ ) to 2014 for oceanic sharks ( $n=18$ species). Light-grey, grey, and dark-grey polygons denote the $50 \%, 80 \%$, and $95 \%$ 2D kernel density estimate of the iterations of LPI vs RFP for the last year (2014). (d) Proportion over time of oceanic sharks with stock assessments that are at a level of biomass or abundance equal or greater than that which would achieve Maximum Sustainable Yield.

370

\section{Extended Data Figure legend:}

372 Extended Data Figure 1. Hierarchical building of the global Living Planet Index and Red 373 List Index.

374 Extended Data Figure 2. (a) Schematic example of constructing the observed (black) and 375 projected (blue) Living Planet Index. First, year-to-year rates of change, abbreviated yyrc 376 thereafter, $\left(d_{t}\right)$ are averaged between species in the same region (e.g., in Region 1, species $A$ 
377 with $d_{A_{t}}$ and species $B$ with $d_{B_{t}}$ averaged in $d_{R 1_{t}}$ ). In a second step, yyrc are averaged

378 between regions Region 1, 2 and 3 to give the global yyrc. The observed LPI builds on yyrc

379 calculated from the estimated abundance index from the state-space population model. The

380 projected LPI builds on yyrc calculated from the estimated and projected abundance index

381 from the state-space population model. Projections are from the last data point to 2020. (b)

382 Global Living Planet Index for oceanic sharks and rays estimated from 1970 to 2018 in black

383 and extrapolated to 2020 in blue. The black and the thick blue lines denote respectively the

384 mean of the estimated and extrapolated LPI. The white and thin blues lines denote

385 respectively, the $95 \%$ credible intervals of the estimated and extrapolated LPI and the grey

386 lines each iteration of the estimated LPI. (c) The annual percentage change was calculated

387 from the posteriors of the estimated LPI (in grey) and extrapolated LPI (in blue) around the

388 final-assessment year relative to the posteriors for 1970. Vertical bars on the 1970-2018

389 period denote the median of the estimated and extrapolated LPI.

390 Extended Data Figure 3. Mean global Living Planet Index (LPI) for oceanic sharks and rays

391 from 1970 to 2018 (black line). Faint gray lines show the effect of excluding all data for a

392 single species at a time and recalculating the mean global LPI for all other species. No means

393 from jackknife species trends fall outside the $95 \%$ credible Interval from the run with all the

394 datasets included, suggesting our selection of species did not unduly influence the overall LPI

395 result.

396 Extended Data Figure 4. Observed (black or empty points, and stars indicate different time-

397 series) and modeled (black line) abundance index for (a) Silky Shark (Carcharhinus

398 falciformis), (b) Oceanic Whitetip Shark (Carcharhinus longimanus), (c) Dusky Shark

399 (Carcharhinus obscurus) and (d) Blue Shark (Prionace glauca) obtained from the state-space

400 population model. The thick black line denotes the mean of the estimated abundance index

401 and the shaded regions denote $95 \%$ credible intervals. 
402 Extended Data Figure 5. Observed (black or empty points, and stars indicate different timeseries) and modeled (black line) abundance index for (a) Scalloped Hammerhead (Sphyrna lewini), (b) Great Hammerhead (Sphyrna mokarran), and (c) Smooth Hammerhead (Sphyrna zygaena) obtained from the state-space population model. The thick black line denotes the mean of the estimated abundance index and the shaded regions denote $95 \%$ credible intervals. Extended Data Figure 6. Observed (points) and modeled (black line) abundance index for

(a) Pelagic Thresher (Alopias pelagicus), (b) Bigeye Thresher (Alopias superciliosus), and (c)

409 Common Thresher (Alopias vulpinus) obtained from the state-space population model. The thick black line denotes the mean of the estimated abundance index and the shaded regions

411 denote $95 \%$ credible intervals.

412 Extended Data Figure 7. Observed (black or empty points, and stars indicate different time-

413 series) and modeled (black line) abundance index for (a) White Shark (Carcharodon

414 carcharias), (b) Shortfin Mako (Isurus oxyrinchus), (c) Longfin Mako (Isurus paucus), and

415 (d) Porbeagle (Lamna nasus) obtained from the state-space population model. The thick

416 black line denotes the mean of the estimated abundance index and the shaded regions denote $41795 \%$ credible intervals.

418 Extended Data Figure 8. Observed (points) and modeled (black line) abundance index for 419 (a) Pelagic Stingray (Pteroplatytrygon violacea), (b) Reef Manta Ray (Mobula alfredi), (c)

420 Giant Manta Ray (Mobula birostris), and (d) Shortfin Devilray (Mobula kuhlii) obtained

421 from the state-space population model. The thick black line denotes the mean of the 422 estimated abundance index and the shaded regions denote $95 \%$ credible intervals.

423 Extended Data Figure 9. (a) Oceanic shark stock status — over time — being at levels of 424 biomass or abundance above Maximum Sustainable Yield (MSY) (green lines) or below 425 MSY (red lines). Dotted lines indicate that a stock is above or below MSY following the last 426 stock assessment value. (b) Number of published stock assessments for oceanic sharks and 
rays over time. (c) Presentation of 14 stocks of oceanic sharks (no available stock

assessments for oceanic rays) status (biomass or abundance over value at MSY) versus pressure $\left(\mathrm{F} / \mathrm{F}_{\mathrm{MSY}}\right)$ in a Kobe plot style, for the last year with available data. Circles represent the species' unique values if only one stock exists and represent the mean of the values of the

431 different stocks (diamonds) when the species has multiple stocks. The plot is divided into

432 four panels: red panel (upper left) with 4 stocks and 3 species, corresponds to stocks that are

433 being overfished and where overfishing is occurring; orange panel (upper right) with 1 stock

434 and 1 species, corresponds to stocks that are not overfished but where overfishing is

435 occurring; yellow panel (bottom left) with 4 stocks and 3 species, corresponds to stocks that

436 are overfished but where overfishing is not occurring; and green panel (bottom right) with 5

437 stocks and 1 species, corresponds to stocks that are not overfished and where overfishing is

438 not occurring.

439 Extended Data Figure 10. Percentage of reported threat categories in the 31 oceanic shark

$440 \quad$ IUCN Red List assessments.

\section{Materials and methods}

443 Data collection and expert selection of oceanic shark time-series

444 Time-series data on relative abundance $(n=57)$ for 18 species (see Supplementary Table S1)

445 were gathered from peer-reviewed publications and the grey literature, including government

446 reports. Relative abundance indices, and associated uncertainty estimates when available,

447 included formal stock assessment outputs (trends in biomass), as well as standardized or

448 nominal catch per unit effort (CPUE) or sightings per unit effort (SPUE) from scientific

449 surveys, fisheries data, or bather protection nets (see Supplementary Table S1 and EDF 4 to

450 8). Entry of original time-series (in the database available at www.sharkipedia.org) was

451 conducted by J.S.Y. and N.K.D. and subsequently independently checked by C.L.R. and N.P. 
452 All datasets underwent extensive checks prior to analyses; their reliability was reviewed and

453 assigned to ocean regions (North, South Atlantic Ocean; Indian Ocean; North, South Pacific

454 Ocean) by experts during an IUCN SSC SSG workshop (Dallas, Texas, USA, 5-9 November

455 2018). Stock assessment outputs were preferred over standardized, then nominal CPUE or

456 SPUE time-series when multiple data sets were available for the same species and region.

457 Stock assessment models integrate the catch history, abundance trends and life-history

458 information to infer population dynamics, whereas CPUE or SPUE represents the trend in

459 relative abundance of the sampled fraction of the population. The details and rationale for the

460 selection of datasets, where pertinent, are presented in the Population section of the relevant

461 Red List assessment (www.iucnredlist.org). Two stock assessments were updated ${ }^{25,51}$ after

462 the workshop and are thus included in our analysis.

463 Data collation and calculation of ecological and life-history traits

464 Estimates of shark age and maximum size can vary regionally, as well as between studies and 465 across regions. Where possible, estimates of generation time (GT) were based on observed 466 rather than theoretical maximum age. Within regions, preference was given to studies that 467 used: validated ages; the widest size range; and, age estimates that included repeat readers, 468 measuring precision, and bias. The validated age estimates from the closest region were used 469 in cases where there was not a published age and growth study for a region, or validated ages 470 from a region ${ }^{52-54}$. Generation time is defined as the median age of parents in the current 471 cohort $^{55}$. Species- and regionally-specific GT (Supplementary Table S1) were calculated 472 from female median age at maturity $\left(A_{\text {mat }}\right)$ and maximum age $\left(A_{\max }\right)$ as $G T=\left(\left(A_{\max }-\right.\right.$ $\left.\left.473 A_{\text {mat }}\right) * z\right)+A_{\text {mat }}$. The constant $z$ depends on the mortality rate of adults and is typically 474 around 0.3 for mammals ${ }^{55,56}$. We chose to assume a more conservative value of $z=0.5$ to 475 account for the likelihood that age structure had already been truncated by overfishing by the 476 time it was measured ${ }^{26,27}$ and that ages of sharks have been systematically underestimated ${ }^{54}$. 
477 The details of GT were presented to the workshop for review and the final choices were used

478 in the published IUCN Red List assessments and associated supplementary material for each

479 species (see also Supplementary Methods 2).

$480 \quad$ Modeling population dynamics

481 To analyze oceanic shark trend data, we used a Bayesian population state-space model

482 designed for IUCN Red List assessments (Just Another Red List Assessment, JARA ${ }^{33,57}$ ),

483 which builds on the Bayesian state-space tool for averaging relative abundance indices by

484 Winker et al. ${ }^{32}$ and is available open-source on GitHub (www.github.com/henning-

485 winker/JARA). Each relative abundance index (or time-series) was assumed to follow an

486 exponential growth defined through the state process equation:

$$
\mu_{t+1}=\mu_{t}+r_{t}
$$

487 where $\mu_{t}$ is the logarithm of the expected abundance in year $t$, and $r_{t}$ is the normally

488 distributed annual rate of change with mean $\hat{r}$, the estimable mean rate of change for a time-

489 series, and process variance $\sigma^{2}$. We linked the logarithm of the observed relative abundance

490 indices to the logarithm of the true expected population trend using the observation equation:

$$
\log \left(y_{t}\right)=\mu_{t}+\varepsilon_{t}
$$

491 where $y_{t}$ denotes the abundance value for year $t, \varepsilon_{t}$ is observation residual for year $t$, which is 492 assumed to be normally distributed on log-scale $\varepsilon_{t} \sim N\left(0, \sigma_{\varepsilon}{ }^{2}\right)$ as a function of the observation 493 variance $\sigma_{\varepsilon}^{2}$.

494 Multiple time-series for a species in a same region (North, South Atlantic Ocean; Indian

495 Ocean; North, South Pacific Ocean) were analysed in a single run and treated as indices

496 following ${ }^{32}$. We used vague normal prior for $\hat{r} \sim N(0,1000)$ and vague inverse-gamma prior

497 for the process variance $\sigma^{2} \sim I G(0.001,0.001)$.

498 For each time-series, we also projected model estimates from the last data point to 2020 to be

499 able to estimate trajectories for the LPI up to the final year of assessment for progress 
500 towards the Aichi Targets. These projections were based on the posteriors of the estimated

501 changes across all years in the observed time-series (see ${ }^{57}$ for details):

$$
\bar{r}=\frac{1}{n} \sum_{t=1}^{n} r_{t}
$$

502 Three Monte Carlo Markov chains were run for each dataset with different initial values.

503 Each Markov chain was initiated by assuming an initial population size in the first year

504 drawn in log-space from a normal distribution with the mean equal to the log of the first

505 available count $\left(y_{1}\right)$ and a standard deviation of 1000 . In each chain, the first 5,000 iterations

506 were discarded ('burn-in'), and of the remaining 50,000 iterations, 10,000 were selected for

507 posterior inference ('thinning rate' $=5$ ). Thus, posterior distributions were estimated from

50830,000 iterations. Convergence of each parameter was checked with the Gelman and Rubin

509 diagnostics ${ }^{58}$. Every model comes with four diagnostic plots: the unscaled input data and

510 uncertainty estimates around each observation in the form $95 \%$ Confidence Intervals, the

511 observed and predicted abundance values for each time-series together with the $95 \%$

512 posterior predictive credibility intervals, individual fits on the log-scale, as well as the $95 \%$

513 Bayesian credible intervals derived from the observation variance, and residual plot (see ${ }^{57}$

514 for detailed description and examples). We conducted posterior predictive checks (drawing

515 simulated values from the joint posterior predictive distribution of replicated data and

516 compare these samples to the observed data) by checking that the credible Interval of the fit

517 of the models fall each time within the posterior predictive distribution limits and that

518 Bayesian p-value were around 0.5 (using Pearson residuals) ${ }^{59,60}$. Analyses were performed

519 using R Statistical Software v3.5.2 $2^{61}$ and via the interface from R ('R2jags' package v0.5-

$5207 ;^{62}$ ) to JAGS ('Just Another Gibbs Sampler' v4.3.0; ${ }^{63}$ ). The Highest Posterior Density

521 interval was used as the interval estimator of $95 \%$ credible intervals.

522 Calculation of Living Planet Index

523 The LPI for oceanic sharks is a quantitative mean index of year-to-year rate of change of all 
524 species that occur in a given region and finally aggregated to a global scale (see EDF 1). The

525 annual rate of change $d_{t}$ for each species in a region is the logarithm of the growth rate of the

526 time-series in a given year $(t)$ :

$$
d_{t}=\log _{10}\left(\frac{I_{t}}{I_{t-1}}\right)
$$

527 where $I_{t}$ denotes the posteriors of the estimated abundance trend in a given year $(t)$ obtained 528 from the Bayesian state-space model outputs.

529 To calculate the global LPI, the annual rates of change $d_{t}$ for each species in a region were 530 then aggregated to provide a single annual rate of change for each region (see EDF 1a for an 531 example), and the same procedure was applied across regions in the same Ocean (if

532 subdivided in south and north regions), and finally across the three Oceans to generate a

533 global year-to-year rate of change. We also computed a global LPI for each species

534 separately, by Oceans and by time-series with similar ecological lifestyle or life-history traits:

535 geographical zone (temperate or tropical), body size (maximum total length), and generation

536 time (following IUCN definition ${ }^{55}$, see Supplementary Table S1). We back-transformed the

$537 \log$ values to the linear scale to generate index values for the range of scales (global, by

538 Ocean, by species or trait-groupings of time-series):

$$
L P I_{t}=L P I_{t-1} \times 10^{\bar{d}_{t}}
$$

539 where $L P I_{t}$ is the Living Planet Index at a given year $(t)$, with $L P I_{t=1}=1$.

540 The global index started in 1970 and was modelled until 2018 using each year-to-year rate of

541 change for the available time-series. In a second step, the global index was extrapolated

542 through to 2020 using each year-to-year rate of change for the available time-series, and their

543 projections after their last data point (see EDF 2a for an example).

544 Although the overall extent of change in the LPI is an indicator of status and trends in

545 biodiversity, the trend may be driven by the data-rich species in our dataset. We evaluated the

546 sensitivity of the LPI to the subset of species, using a jackknife procedure in which we 
547 sequentially dropped individual species and recalculated the index (see EDF 3).

548 Calculation of Relative Fishing Pressure

549 To investigate the underlying drivers of the abundance trend decline, we calculated the

550 Relative Fishing Pressure, the changes in catch from 1970 to 2014 (end of the available data),

551 relative to abundance (LPI) over the same time period, and scaled by the Relative Fishing

552 Pressure in 1970. First, we extracted the total Sea Around Us Project reconstructed reported

553 and unreported catch data ${ }^{64}$ by species for 14 of our 18 focal species - catch data were not

554 available for 4 of the species: A. pelagicus, Reef Manta Ray M. alfredi, Shortfin Devilray M.

555 kuhlii, Pelagic Stingray P. violacea, and thus were not included in this analysis. To account

556 for the disproportionately high catch of some species (e.g., Blue Shark) in the total catch that

557 could affect the overall pattern, we scaled the catch data at the species level ( $s p)$ to the first

558 catch value in each time-series before summing across species. The Relative Fishing Pressure

559 (RFP) was then calculated as:

$$
R F P_{t}=\frac{\frac{\sum_{s p} \operatorname{catch}_{t}}{L P I_{t}}}{\frac{\sum_{s p} \operatorname{catch}_{t=1970}}{L P I_{t=1970}}}
$$

560 with $L P I_{t}$ being the LPI of the 18 oceanic sharks in year $t$. We also calculated the RFP with

561 the $L P I_{t}$ of only the 14 species for which catch data were available and this was not credibly

562 different from the RFP for all 18 species.

563 Calculation of Red List Index

564 We calculated the RLI based on the proportion of the 31 oceanic shark species in each IUCN

565 Red List category in 1980, 2005, and 2018 (see Supplementary Table S2). The categories

566 used in the assessments were Critically Endangered (CR), Endangered (EN), Vulnerable

567 (VU), Near Threatened (NT), and Least Concern (LC). No species of oceanic shark were

568 assessed in the categories Extinct (EX), Extinct in the Wild (EW), or Not Evaluated (NE).

569 The statuses in 2018 were assigned by the IUCN SSC SSG (Dallas, Texas, USA, 5-9 
570 November 2018). For the RLI of 2005, we used the assessments published between 2000 and

571 2010. Red List assessments for 2005 and 2018 are published on the IUCN Red List of

572 Threatened Species website ${ }^{65}$. Following the recommended IUCN methodology, species

573 previously assessed as Data Deficient (DD) were retrospectively assigned a data-sufficient

574 category (see Table S2). No assessment was available in the 1980s and experts involved in

575 the IUCN SSC SSG workshop (Dallas, Texas, USA, 5-9 November 2018) retrospectively

576 determined Red List statuses for 1980, as well as missing statuses in $\sim 2005$, as per ${ }^{31}$. To

577 account for uncertainty around a retrospective assessment, we used a bootstrap-like method

578 to iteratively resample 10,000 times each species' status from its retrospective assigned status

579 or one category better, or one category worse, denoted by the error bar (the range of

580 bootstrap-like results) in Fig. 3a around the retrospective RLI in 1980 (black dot).

581 The RLI value of a particular year $(t)$ is calculated by multiplying the number of species $(s)$ in

582 each Red List category by the category weight $(W)$ ( 0 for LC, 1 for NT, 2 for VU, 3 for EN, 4

583 for $\mathrm{CR}$, and 5 for EX), then summing the product and dividing by the maximum possible

584 product (number of species $(N)$ multiplied by the maximum weight 5), and subtracted from 1

585 to have an index between 0 (where all species are EX) and 1 (where all species are LC) ${ }^{31}$ :

$$
R L I_{t}=1-\frac{\sum_{s} W_{c(t, s)}}{W_{E X} * N}
$$

586 To make the RLI in 2018 spatially explicit, we calculated $100,000 \mathrm{~km}^{2}$ hexagonal cells in the

587 world's oceans ${ }^{66}$ using the IUCN Red List status of species that are distributed in each unique

588 cell (based on IUCN distribution maps for each species, see Red List assessments). We

589 analyse the difference of RLI between 1980 and 2018 in the same way, assuming the

590 distribution of species did not change in between those years. All spatial data described were

591 processed using ESRI ArcGIS v10.7 $7^{67}$ and R Statistical Software v3.5.2 ${ }^{61}$ in Eckert IV equal-

592 area Projection.

593 The stand-alone point labelled 'Global sharks' in Figure 3a indicates the starting point for the 
594 global chondrichthyan (sharks, rays, and chimaeras) Red List Index calculated from the Red

595 List status as reported in 2006 (the median date of available Red List assessments at this

596 time $^{16}$.

$597 \quad$ Sustainability of stocks of oceanic sharks

598 In order to represent the status of stocks (populations) of oceanic sharks, we compiled total

599 biomass or abundance, relative to Maximum Sustainable Yield (MSY), provided by authors

600 or extracted from the latest available stock assessment reports (the reference of the source and

601 the trajectory used are in Supplementary Table S3). A stock assessment is the process of

602 employing statistical models to quantify the population dynamics of a fished stock in

603 response to fishing based on the best available catch, abundance, and life-history information.

604 No stock assessment exists for any of the oceanic rays and one of the Blue Shark stock

605 assessments could not be included because no estimates of MSY-related quantities were

606 available ${ }^{68}$. We thus used the eight species (Oceanic Whitetip Shark, Dusky Shark, Shortfin

607 Mako, Porbeagle, Scalloped Hammerhead, Great Hammerhead, Smooth Hammerhead, and

608 Blue Shark) with published biomass or abundance trajectories relative to MSY (15 stocks in

609 total) to produce the global proportion - over time - that these species were at levels above

610 the biomass or abundance achieving the MSY (i.e., $p\left(\mathrm{~B}>\mathrm{B}_{\mathrm{MSY}}\right)$ ), and thus not overfished

611 (Figure 4d). Each stock's biomass or abundance relative to MSY was transformed into a

612 binary variable, indicating if the stock was above (1) or below (0) MSY. To represent the

613 status of species with several stocks, we calculated the proportion - over time - of stocks

614 above or below MSY. We then calculated the global proportion - over time - that these

615 species were at levels above the biomass or abundance achieving the MSY by averaging

616 species' status proportion that were above MSY for each year.

617 In a stock assessment, scientists attempt to estimate the amount of fishing mortality (F) over

618 time, and the fishing mortality that will achieve MSY ( $\left.\mathrm{F}_{\mathrm{MSY}}\right)$. Using available stock 
619 assessments, we compiled the latest value of fishing mortality relative to the fishing mortality 620 at MSY (F/ $\left.\mathrm{F}_{\mathrm{MSY}}\right)$ and plotted them against the latest value of biomass or abundance

621 trajectories relative to the MSY, in the 'four quadrant, red-yellow-green' Kobe plot style $622($ EDF 9c).

623

624 Additional References:

625 51. Tremblay-Boyer, L., Carvalho, F., Neubauer, P. \& Pilling, G. Stock assessment for oceanic whitetip shark in 626 the Western and Central Pacific Ocean. Rep. WCPFC Sci. Comm. Fifteenth Regul. Sess. 12-20 August 2018 627 Pohnpei Fed. States Micrones. 98 (2019).

628 52. Cailliet, G. M. \& Goldman, K. J. Age determination and validation in chondrichthyan fishes. in Biology of 629 sharks and their relatives 404-453 (CRC press, 2004).

630 53. Cailliet, G. M. Perspectives on elasmobranch life history studies: a focus on age validation and relevance to 631 fishery management. J. Fish Biol. 87, 1271-1292 (2015).

632 54. Harry, A. V. Evidence for systemic age underestimation in shark and ray ageing studies. Fish Fish. 19, 185$633200(2018)$.

634 55. IUCN Standards and Petitions Subcommittee. Guidelines for using the IUCN Red List Categories and

635 Criteria. Version 13. Prep. Stand. Petitions Subcomm. Gland Switz. Camb. UK IUCN (2017).

636 56. Pacifici, M. et al. Generation length for mammals. Nat. Conserv. 5, 89 (2013).

637 57. Winker, H., Pacoureau, N. \& Sherley, R. B. JARA: 'Just Another Red-List Assessment'. bioRxiv 672899

638 (2020) doi:10.1101/672899.

639 58. Gelman, A. \& Rubin, D. B. Inference from iterative simulation using multiple sequences. Stat. Sci. 7, 457$640 \quad 472(1992)$

641 59. Conn, P. B., Johnson, D. S., Williams, P. J., Melin, S. R. \& Hooten, M. B. A guide to Bayesian model

642 checking for ecologists. Ecol. Monogr. 88, 526-542 (2018).

643 60. Gelman, A. et al. Bayesian data analysis. (CRC press, 2013).

644 61. R Core Team. R: A language and environment for statistical computing. $R$ Foundation for Statistical 645 Computing, Vienna, Austria (2018).

646 62. Su, Y.-S. \& Yajima, M. R2jags: Using R to run 'JAGS'. R Package Version 05-7 34, (2015).

647 63. Plummer, M. JAGS version 4.3. 0 user manual. (2017). 
64. Pauly, D. \& Zeller, D. Sea Around Us concepts, design and data. Vanc. BC (2015).

65. IUCN. The IUCN Red List of Threatened Species. Version 2019-2. http://www.iucnredlist.org (2019).

66. Natural Earth. 10m ocean coastlines. Retrieved October, 27, 2019, from

https://www.naturalearthdata.com/downloads/10m-physical-vectors/10m-ocean/. (2019).

67. Environmental Systems Research Institute (ESRI). ArcGIS Desktop: Release 10.7.1. Redlands, CA (2019).

68. Takeuchi, Y., Tremblay-Boyer, L., Pilling, M. \& Hampton, J. Assessment of blue shark in the southwestern

Acknowledgments: We thank all members of the IUCN Species Survival Commission Shark

657 Specialist Group and other experts who contributed to the data collation. In particular, we 658 extend our gratitude to Alexandre Aires-da-Silva, Felipe Carvalho, Jessica Cheok, Shelley

659 Clarke, Rui Coelho, Enric Cortés, Trey Driggers, Christine Dudgeon, Mike Hoffmann, Yan

660 Jiao, Tom Kashiwagi, Alison Kock, Chris Lowe, Joel Rice, Laura Tremblay-Boyer, Wade J.

661 VanderWright, and Sabine Wintner. The scientific results and conclusions, as well as any

662 views or opinions expressed herein, are those of the author(s) and do not necessarily reflect

663 those of institutions or data providers. This project was funded by the Shark Conservation

664 Fund, a philanthropic collaborative pooling expertise and resources to meet the threats facing

665 the world's sharks and rays. The Shark Conservation Fund is a project of Rockefeller

666 Philanthropy Advisors. This work was funded by the Shark Conservation Fund as part of the

667 Global Shark Trends Project to N.K.D. and C.A.S., and US National Science Foundation

668 grant DEB-1556779 to H.K.K. P.M.K. was supported by the Marine Biodiversity Hub, a

669 collaborative partnership supported through funding from the Australian Government's

670 National Environmental Science Program. N.K.D. was supported by a Natural Science and

671 Engineering Research Council Discovery and Accelerator Awards and the Canada Research

672 Chairs Program. 
673 Author Contributions: C.L.R., P.M.K., R.A.P., and N.K.D. organized and led the workshop 674 investigation of data quality and facilitated the 2018 Red List assessments. N.P., H.K.K., and 675 N.K.D. conceptualized the analysis. J.S.Y., C.L.R., H.K.K., R.B.S., N.P., and N.K.D. 676 compiled and curated the time-series data. J.K.C., A.M., and H.W. provided additional time677 series data. N.P., R.B.S., and H.W. conducted the statistical analysis. N.P., H.K.K., and 678 N.K.D. visualized the data and wrote the first draft. N.K.D. and H.K.K. acquired the funding. 679 All authors discussed time-series, the analysis and results, and contributed to writing the 680 manuscript.

681 Authors declare no competing interests.

682 Author Information: * Correspondence and requests for materials should be addressed to 683 n.pacoureau@gmail.com; r.sherley@exeter.ac.uk.

684 Supplementary Information is linked to the online version of the paper at 685 www.nature.com/nature.

686 Reprints and permissions information is available at www.nature.com/reprints.

687 Data and materials availability: Data are available on www.sharkipedia.org and at 688 https://zenodo.org/badge/latestdoi/307472870. 


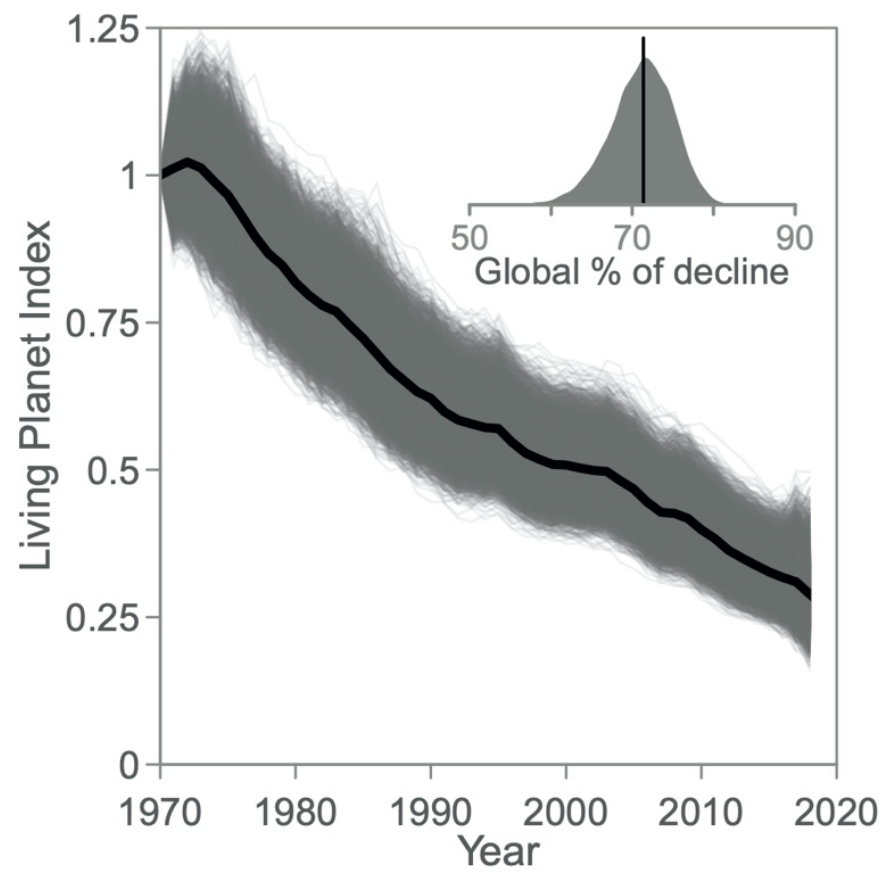

Figure 1. Global Living Planet Index (LPI) for 18 oceanic sharks estimated from 1970 to 2018. The global percentage (\%) of decline was calculated from the posteriors of the LPI around the final assessment year relative to the posteriors for 1970. The black line denotes the mean, the white lines the $95 \%$ credible intervals and the grey lines each iteration.
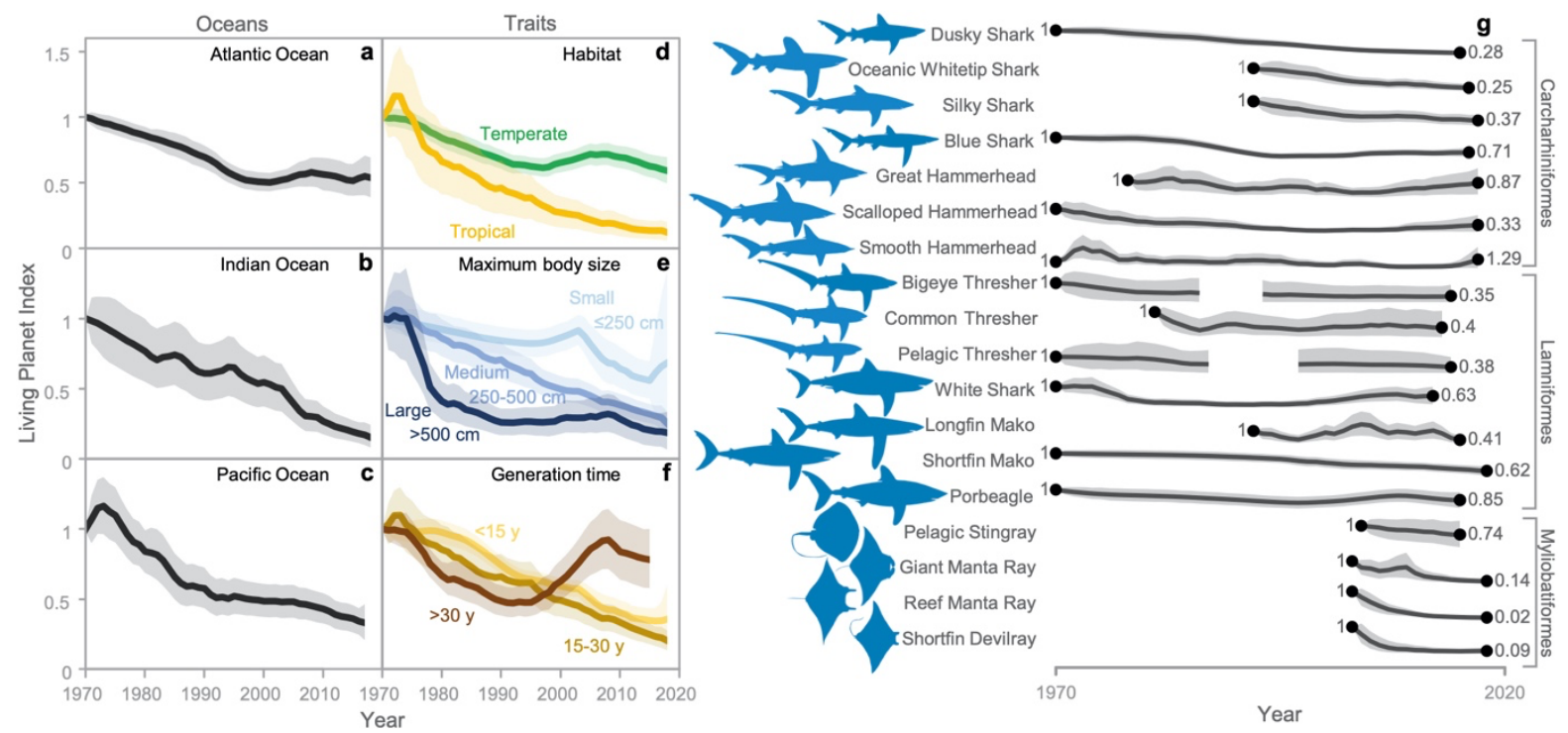

Figure 2. Living Planet Index for 18 oceanic sharks from 1970 to 2018 disaggregated by Oceans (a, b, c), and the traits (d) geographical zone, (e) body size (maximum total length), (f) generation time (GT), and (g) species (species' time-series are in Extended Data Figure 4 to 8). Lines denote the mean and shaded regions the $95 \%$ credible intervals. 

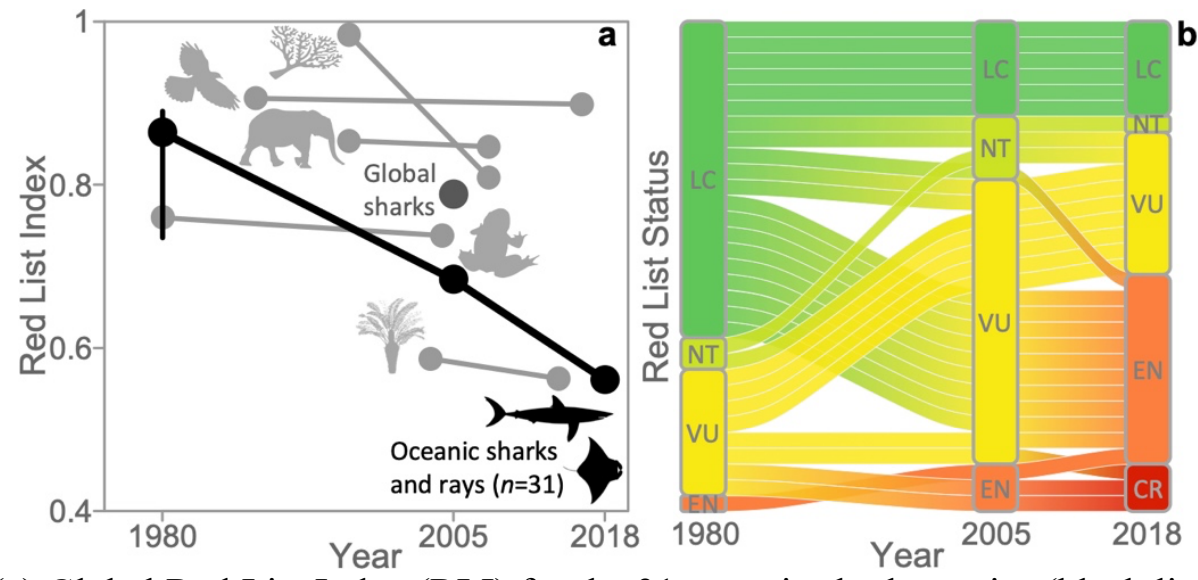

Figure 3. (a) Global Red List Index (RLI) for the 31 oceanic shark species (black line) estimated in 1980, 2005, and 2018, and for mammals, birds, amphibians, reef-forming corals, and cycads (in grey), and global chondrichthyans (sharks, rays, and chimaeras; point labelled 'Global sharks') ${ }^{16}$. The error bar denotes the uncertainty around the retrospective 1980 IUCN status (see Methods). A RLI value of 1.0 equates to all species qualifying as Least Concern (i.e., not expected to become Extinct in the near future), while a RLI value of 0 equates to all species having gone Extinct. (b) Change in Red List status of oceanic sharks from 1980 to 2018.
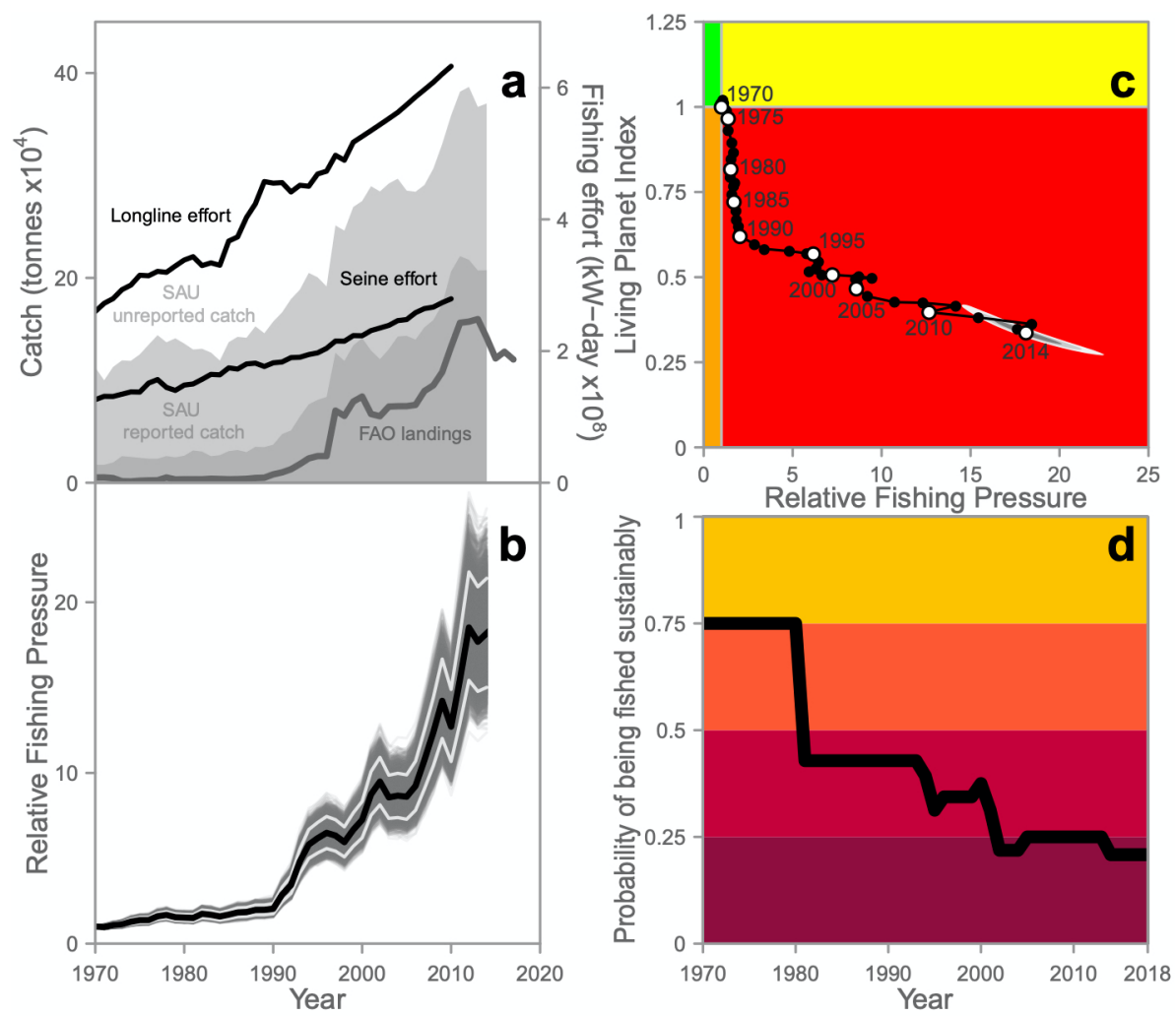

Figure 4. (a) Global catch data of 14 oceanic sharks and fishing effort of longline and seine gears. SAU: Sea Around Us project. FAO: Food and Agriculture Organization of the United Nations. Longline and Seine effort are effective corrected fishing effort ${ }^{36}$. (b) Fishing pressure (catch) encountered by oceanic sharks relative to the fishing pressure (catch) in 1970 and to their abundance from 1970 to 2014 . The black line denotes the mean, the white lines the $95 \%$ credible intervals and the grey lines each iteration. (c) Living Planet Index (LPI) as a function of Relative Fishing Pressure (RFP, $n=14$ species) from 1970 (the initial state where LPI and RFP $=1$ ) to 2014 for oceanic sharks ( $\mathrm{n}=18$ species). Light-grey, grey, and dark-grey polygons denote the $50 \%, 80 \%$, and 95\% 2D kernel density estimate of the iterations of LPI vs RFP for the last year (2014). (d) 
Proportion over time of oceanic sharks with stock assessments that are at a level of biomass or abundance equal or greater than that which would achieve Maximum Sustainable Yield. 